Classification

Physics Abstracts

73.60

\title{
Dispersion of the Valence Electron Energy Loss in Thin Amorphous Carbon Films deposited by Ion Assisted Evaporation of Graphite
}

\author{
Uwe Falke, Anne-Katrin Weber and Jens Ullmann \\ TU Chemnitz-Zwickau, Institut für Physik, D-09107 Chemnitz, Germany
}

(Received September 29; accepted December 20, 1994)

\begin{abstract}
The dispersion of electron energy loss with transferred wave vector $q$ has been measured for amorphous, non-hydrogenated carbon films. The peak energies for both the loss peak related to the main plasmon loss and for that brought about by the $\pi \rightarrow \pi^{*}$ interband transitions are found to follow a power law in $q$. The films exhibited differences of the exponents which may be related to structural differences. For the material deposited with very small ion flux, which is quite similar to ordinary evaporated carbon, a dispersion of the two low loss peaks with $q^{1.6}$ and $q^{1.9}$, respectively, has been found whereas in the case of the other two films, the dispersion appears to be nearly linear in $q$.
\end{abstract}

\section{Introduction}

Electron energy-loss spectroscopy (EELS) has been used widely to investigate amorphous carbon films [1]-[12]. The EELS spectrum provides information about the electronic structure. The conservation of energy and momentum, $\hbar q$, in the inelastic scattering enables the investigator to measure the $q$ dispersion of the interesting features by taking angle resolved spectra. Measurements of the $q$-dependence of the valence energy loss in carbon materials have been carried out by Fink et al. [13] on polymers. Other researchers examined the single electron excitation which is an approach to the momentum distribution of the electrons in the specimen [14].

The electronic density of states (DOS) of amorphous carbon has few features due to the lack of long range order and the integration of energy losses from sites having different surroundings. Only two distinguishable peaks appear in the low loss range between 1 and $50 \mathrm{eV}$. The first one is located at $(4 \ldots 7) \mathrm{eV}$ and arises from interband transitions of the $\pi$ electrons to $\pi^{*}$ states. The second one is centered at $(20 \ldots 33) \mathrm{eV}$, dependending on the material, and is regarded as the freeelectron plasmon excitation. Although this point of view should be problematic because of the $\sigma \rightarrow \sigma^{*}$ interband transitions at approximately $12-16 \mathrm{eV}$ which modify the Drude loss function [15], the free-electron plasmon model has proved to be a sufficient description of this loss peak representing the total valence electron density $[4,5,16]$. 


\section{Experimental}

The carbon films were deposited onto Si by an ion assisted electron beam evaporation of graphite (IAE, [17]). This method involves a bombardment of the condensing material ${ }^{1}$ with noble gas ions (here: $\mathrm{Ne}^{+}$) having energies in the range $\left(10^{2} \ldots 10^{3}\right) \mathrm{eV}$. The ion energy and the ratio of ions to neutral particles arriving at the substrate strongly influence the film properties (see Tab. I). From high resolution transmission electron microscopy (HRTEM) and electron diffraction it was found that sample 1 is fully amorphous. The other samples exhibit a turbostratic structure, most pronounced in sample 3. From the near edge fine structure (ELNES) of the CK loss edge the $\mathrm{sp}^{3}$ content of the films was estimated according to the procedure proposed in [3], see [17].

Table I. - Deposition parameters of the investigated films and content of $s^{3}$ hybridized carbon atoms as determined by analysis of $C-K E L N E S$.

\begin{tabular}{||l||c|c|c||}
\hline \hline & $\begin{array}{l}\text { ratio of ion and neutral } \\
\text { particle fluxes } V_{\mathrm{i} / \mathrm{n}}\end{array}$ & ion energy $E_{\text {ion }} / \mathrm{eV}$ & $\mathrm{sp}^{3}$ content $/ \%$ \\
\hline \hline sample 1 & 0.1 & 200 & 0 \\
\hline sample 2 & 2 & 100 & 30 \\
\hline sample 3 & 2 & 600 & 20 \\
\hline \hline
\end{tabular}

The remarkable content of $\mathrm{sp}^{3}$ hybridized carbon in films 2 and 3 is due to the influence of the noble gas ions during film deposition. McKenzie [18] attributes the very high amounts of $\mathrm{sp}^{3}$ hybridization in carbon deposition methods using carbon ions, such as cathodic arc deposition, to the same mechanism, which has been named subplantation effect. The impinging ions deposite their energy in a very small volume. In a certain energy range the tetrahedral bonded carbon phase is favoured. A further increase of the ion energy leads to the relaxation of this metastable phase, which results in graphitic short range order in the material. The variation of the orientations of these small graphitic areas is the reason for the evolution of a turbostratic material.

The deposited films were floated off the substrate by use of a mixture of $\mathrm{HF}: \mathrm{HNO}_{3}$. Then the films were washed in distilled water and picked up onto TEM copper grids.

The EELS measurements were carried out in a Philips CM20FEG transmission electron microscope, equipped with a GATAN 666 PEELS-spectrometer, in the diffraction mode ("image coupled").

Selection of the scattering angles at which spectra were taken was done by tilting the incident beam using the dark field deflection unit. The tilt has been calibrated by reference of a thin polycrystalline Al-film.

The integration time and spectrometer entrance aperture were varied within each series to compensate for the decay of the inelastic cross section at larger scattering angles. The accelerating voltage was $80 \mathrm{kV}$; the energy resolution determined as the FWHM of the zero loss peak was not worse than $0.9 \mathrm{eV}$. The $q$ resolution was $0.025 \AA^{-1}$ at smaller scattering angles and $0.05 \AA^{-1}$ for $q>0.6 \AA^{-1}$. 


\section{Results}

The spectra were recorded for scattering angles related to a range of $q$ between $0.12 \AA^{-1}$ and $1.00 \AA^{-1}$. The EELS spectrum at the mean scattering angle 0 extends over such a large dynamic range that the $\mathrm{PD}$ array of the spectrometer was overloaded heavily in the range of the zero loss peak whereas in the plasmon loss range only a few tens of counts were recorded per read-out sequence. For this reason those data were left out.
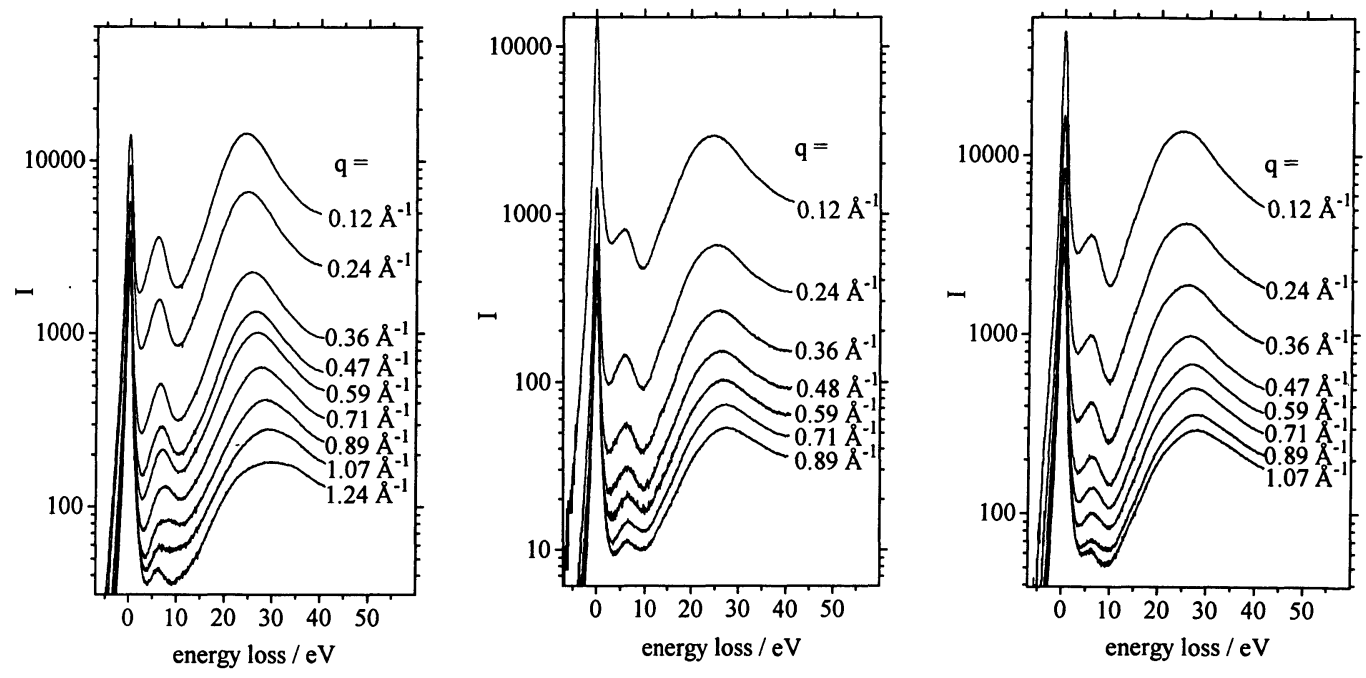

Fig. 1. - Measured spectra (left: sample 1, middle: sample 2, right: sample 3).

The raw data are displayed in Figure 1. With increasing scattering angle, a shift of the two distinctive peaks to higher energy losses can be seen. Mixed large angle elastic and small angle inelastic scattering contributes to the spectra especially at higher $q$ [19]. This effect leads to a replication of the loss profiles characteristic for small $q$ values at $q>0.8 \AA^{-1}$. The elastic scattering contribution consists of a Bragg scattering part and a more spread-out quasielastic phonon scattering part. Elastic and quasielastic scattering may be treated as one, since the related energy losses are much smaller than the energy resolution of the measurement. The quasielastic scattered electrons are distributed very smoothly over the whole angle range of interest. The Bragg scattering concentrates in certain scattering solid angles depending on most likely distances and orientations of bondings. It is much weaker in amorphous materials than in crystalline, but it is not fully negligible. As the electron diffraction patterns for the films show, a maximum of diffracted intensity occurs at scattering angles related to the interplanar spacing in the turbostratic material. As a rough estimation, this spacing can be regarded as equal to the (0002) spacing in graphite, which is $3.35 \AA$. So we find $q=1.9 \AA^{-1}$. Keeping in mind the large intensity ratio between the unscattered beam and this first Bragg maximum in our case, one may neglect this contribution to the recorded spectra. Then, only the quasielastic large angle scattering mixed with inelastic scattering remains. In [19] it was shown that a spectrum recorded from a sufficiently large collection angle range represents this mixed scattering very well. Another approach to correct for this scattering contribution is to reconstruct such an angle integrated spectrum by suitable summation of the angle-resolved spectra. The spectral shape of the mixed quasi-elastic and inelastic scattering can 


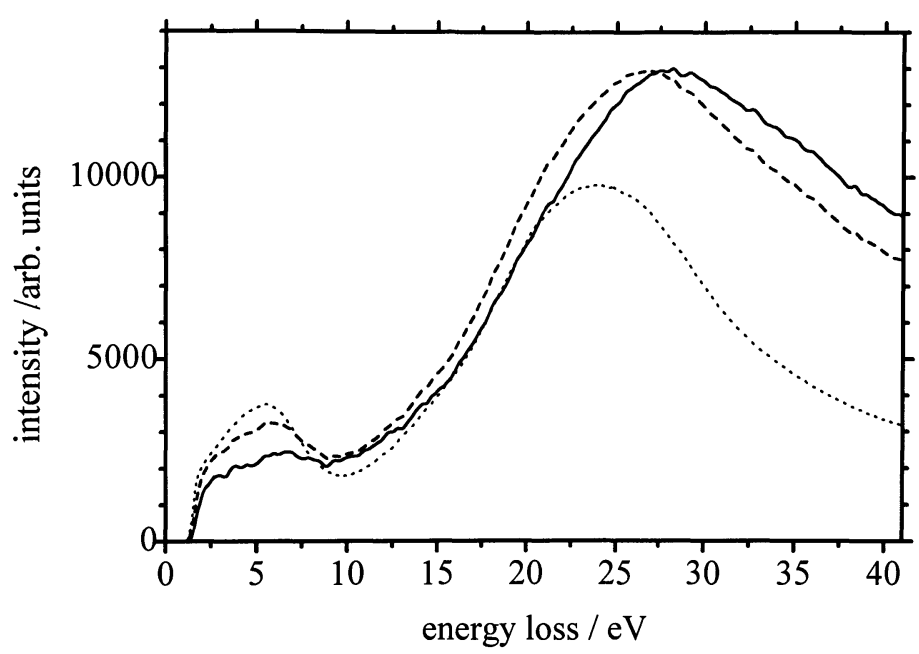

Fig. 2. - Removal of calculated mixed elastic and ineleastic scattering from high angle loss data dashed: spectrum measured at $q=1.24 \AA^{-1}$, dotted: mixed quasielastic-inelastic scattering $M(E)$ after (1), solid: corrected spectrum. The curves are not to scale, expecially $M$ is enlarged.

be approximated by calculating the integral

$$
M(E)=C \int_{0}^{\theta_{\max }} \frac{1}{q^{2}} \operatorname{Im}\left(-\frac{1}{\epsilon}\right) \mathrm{d} \theta
$$

where the upper integration limit is choosen in such a way, that the contributions of the integrand at all larger angles are negligible and the loss function may be regarded as independent on the scattering angle within the integration range. As known, the most probable scattering angle for the inelastic scattering is $\theta_{E}$ [20]. This angle is very small for the plasmon loss range: with 20 $\mathrm{eV}$ energy loss and $80 \mathrm{keV}$ primary energy a $\theta_{E}$ of 0.12 mrad can be calculated, corresponding to $q=0.03 \AA^{-1}$. The energy loss spectrum at such small angles is influenced mostly by the $1 / q^{2}$ dependence of the inelastic scattering cross section rather then by the $q$ dependence of the loss function. Therefore, the integrand may be calculated using energy-loss data recorded at small scattering angles. However, the determination of the absolute intensity of the term $M(E)$ in a recorded spectrum is not exactly possible without knowledge about the full spectrum in the scattering solid angle around 0 including the zero loss peak. Therefore, only an example of the effect of the described correction is given in Figure 2. The mixed-scattering part of the measured spectrum was estimated so that the resulting corrected spectrum fits in the $q$-series established from lower- $q$ spectra, in which mixed scattering may be neglected.

To proceed extracting the loss functions from the measured spectra, firstly, the zero loss peak was removed. This was done by mirroring the high energy tail of the zero loss peak into the loss range between 0.5 and $4 \mathrm{eV}$. The mirrored intensity was subtracted. In the range between $2 \mathrm{eV}$ and $4 \mathrm{eV}$ an exponential fit to the mirrored zero loss peak was done to extrapolate the zero loss peak tail to higher loss energies. This tail arises mainly from the so-called point spread function (PSF) of the photodiode array (PDA). The extrapolation was subtracted as well then. This procedure provides reasonably good results since the zero loss peak shape on the device used is nearly symmetric. From $1.5 \mathrm{eV}$ energy loss downward to zero the spectra were extrapolated by power law functions. Twofold inelastic scattering was roughly corrected using the method 

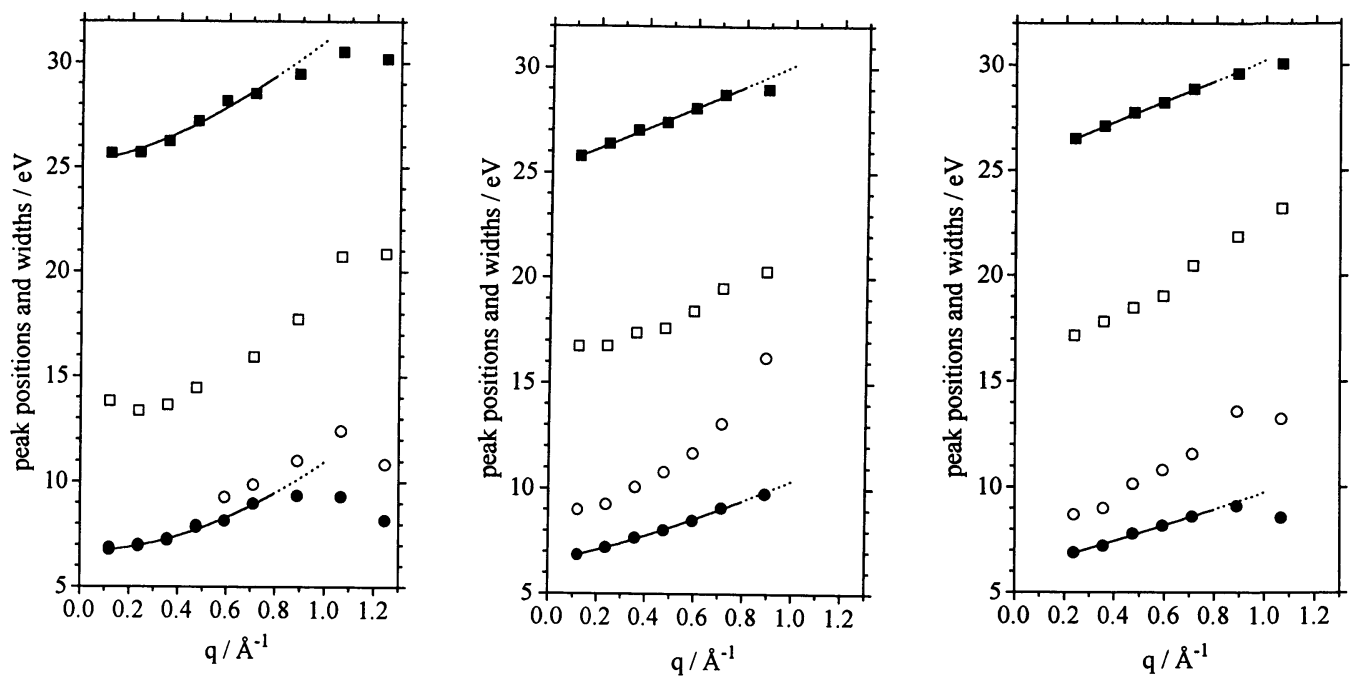

Fig. 3. - Positions and widths of the two peaks of the loss function. Full symbols: peak positions, hollow symbols: peak widths; squares: main plasmon peak, circles $\pi \rightarrow \pi^{*}$ peak.
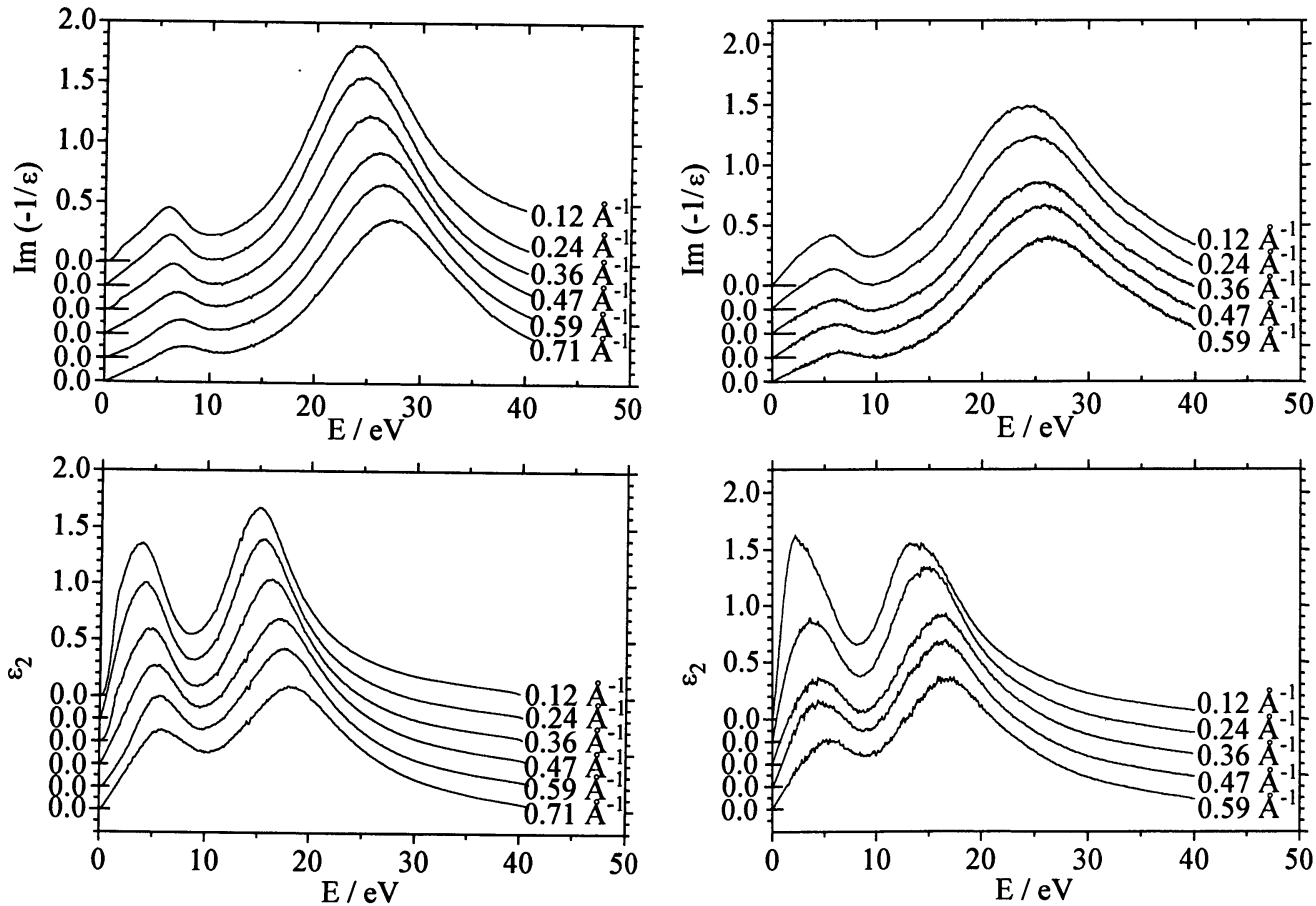

Fig. 4. - Loss functions and the imaginary parts of the dielectric function of sample 1 (left) and sample 2 (right).

described by Daniels et al. [21]. This method provides no angle dependent convolution, which must be applied to treat the problem the exact way. Nevertheless, the multiple loss signal in the spectrum is relatively small in the loss range of interest below $35 \mathrm{eV}$, and the change of the loss probability with the scattering angle is not as dramatic. Therefore, the extracted single loss curves 
should be reliable up to the main plasmon peak and somewhat beyond.

These curves differ from the energy loss functions only by constant factors. The normalization was done using the sum rule [15]

$$
\int_{0}^{\infty} \omega \operatorname{Im}\left(-\frac{1}{\epsilon(\omega)}\right) \mathrm{d} \omega=\frac{\pi}{2} \omega_{\mathrm{p}}^{2}
$$

Use of this relation has the advantage that no additional data, such as refractive index, must be known. The plasmon frequency may be extracted from the EELS data. It must be stated however, that the maximum of the plasmon loss peak is shifted relative to the plasmon energy, $\hbar \omega_{\mathrm{p}}$, due to the plasmon damping. In this work, the plasmon energy is derived by fitting a Drude loss function to the measured loss function at the lowest scattering angle.

The positions of the $\pi \rightarrow \pi^{*}$ interband transition peak and of the main plasmon peak were determined by fits of Lorentzians to the loss functions. The measured peak positions and peak widths are shown in Figure 3. Functions of the form $A+B q^{\mathrm{C}}$ were fitted to the measured peak positions in order to quantify the differences between the samples. The results are given in Table II. The deviations of the obtained curves from power law above $0.8 \AA^{-1}$ seems to be due to the effect of mixed scattering as discussed above. Therefore, only the data up to $0.71 \AA^{-1}$ were used for the fit.

For the loss functions and the imaginary parts of the dielectric functions of films 1 and 2, see Figure 4.

Table II. - Fits of the measured dependence of the loss peak positions on the transferred wave vector $q$ to $A+B q^{\mathrm{C}}\left(q\right.$ in $\left.\stackrel{\circ}{ }^{-1}\right)$.

\begin{tabular}{||c||c|c|c|c|c|c||}
\hline \hline \multirow{2}{*}{ sample } & \multicolumn{3}{c|}{ position of $\pi \rightarrow \pi^{*}$ peak } & \multicolumn{3}{c||}{ position of main plasmon peak } \\
\cline { 2 - 7 } & $A / \mathrm{eV}$ & $B / \mathrm{eV}$ & $C$ & $A / \mathrm{eV}$ & $B / \mathrm{eV}$ & $C$ \\
\hline 1 & 6.7 & 4.3 & 1.9 & 25.3 & 5.9 & 1.6 \\
\hline 2 & 6.6 & 3.8 & 1.3 & 25.3 & 4.9 & 1.1 \\
\hline 3 & 6.1 & 3.7 & 1.1 & 25.3 & 5.0 & 1.0 \\
\hline \hline
\end{tabular}

\section{Discussion}

The dielectric function exhibits two broad transition bands at approximately (4...5) eV and (12...16) $\mathrm{eV}$. These are related to the $\pi \rightarrow \pi^{*}$ and $\sigma \rightarrow \sigma^{*}$-transitions. In the case of an other amorphous material, namely silicon, it was shown [23], that, in the same way as the densities of states $N(E)$, the spectral functions $\eta(E, k)$ correspond to the smeared out band structure of the crystalline material. Here, the short range order (SRO) of the films is graphitic, due to the predominant $\mathrm{sp}^{2}$-hybridization in the a-C films, which had been estimated by the investigation of the C-K near edge structure [17]. This is supported by the similarity of the essential features of the measured data to those of graphite [22], bearing in mind the amorphous nature of the films.

The EELS data of the investigated layers exhibit differences between film 1 on the one hand and films 2 und 3 on the other. The $\pi \rightarrow \pi^{*}$ peak and the main plasmon loss peak are more narrow in sample 1 . The peak widths represent the life times of the related collective excitations. The shorter 
life times in the turbostratic samples 2 and 3 may arise from the higher degree of inhomogenity there. We suppose the existence of clusters with graphitic SRO which are connected by $\mathrm{sp}^{3}$ bonds. The collective excitations may therefore be damped due to size effects then.

The measured peak dispersion deviates from a quadratic slope, which is expected for plasmon excitations [15], for all three samples. We believe, that the interaction between the dispersion of interband transitions and that of the collective excitations is responsible for this behaviour. The dispersion exponents reflect the structural differences of the films, that is, the increasing turbostratic character going from sample 1 to 3.

In principle, the dispersion of the energy loss contains information about the k-dependence of the electron density of states, expressed by the spectral functions. However, the relevance of the joint density of states complicates the approach to the density-of-states functions or the spectral functions respectively, since the deconvolution procedure would be very critical due to the very smooth shape of the functions and the inevitable errors included in each measurement. Additionally, the experimental data normally average over a large volume of the investigated material. Such a volume contains a lot of at least slightly different networks or clusters of the size proposed in recent theoretical models, resulting in an additional broadening of all features in the loss data. Nevertheless, it turns out that not only the usually considered EELS informations, such as the near edge structure or the loss function at $q \approx 0$, but also the dispersion of the energy loss with $q$ may characterize differences between amorphous structures.

\section{Acknowledgements}

The authors wish to thank S. Schulze for constructive discussion. One of us (J.U.) would like to notice financial support by the Deutsche Forschungsgemeinschaft (DFG, project number JU 117/5-1) provided under the auspices otf the trinational "D-A-CH" German, Austrian and Swiss cooperation on the synthesis of superhard materials.

\section{References}

[1] Fink J., Müller-Heinzerling Th., Pflüger J., Scheerer B., Dischler B., Koidl P., Bubenzer A. and Sah R.E., Phys. Rev. B 30 (1984) 4713.

[2] Richter A., Scheibe H.J., Pompe W., Brzezinka K.W. and Mühling I., J. Non-Cryst. Solids. 88 (1986) 131-144.

[3] Berger S.D., McKenzie D.R. and Martin P.J., Phil. Mag. Lett. 57 (1988) 285-290.

[4] Cuomo J.J., Doyle J.P., Bruley J. and Liu J.C., J. Vac. Sci. Technol. A. 9 (1991) 2210-2215.

[5] Cuomo J.J., Bruley J., Doyle J.P., Pappas D.L., Saenger K.L., Liu J.C. and Batson P.E., Mat. Res. Soc. Proc. 202 (1991) 247-252.

[6] Rother B., Siegel J., Kappich K.-U. and Breuer K., Diamond Related Mater. 1 (1992) 865-867.

[7] Bounouh Y., Theye M.L., Dehbi-Alaoui A., Matthews A., Cernogora J., FaveJ.L., Colliex C., Gheorghiu A. and Senemaud C., Diamond Related Mater. 2 (1993) 259- 265.

[8] Veerasamy V.S., Amaratunga G.A.J., Milne W.I., Hewitt P., Fallon P.J., McKenzie D.R. and Davis C.A., Diamond Related Mater. 2 (1993) 782-787.

[9] Maydell E.A.,Dunlop E., Fabian D.J., Haupt J. and Gissler W., Diamond Related Mater. 2 (1993) 873878.

[10] Fallon P.J., Veerasamy V.S., Davis C.A., Robertson J., Amaratunga G.A.J., Milne W.I. andKoskinen J., Phys. Rev. B 48 (1993) 4777-4782. 
[11] Scheibe H.-J. and Schultrich B., Thin Solid Films 246 (1994) 92-102.

[12] Andre B., Rossi F., van Deen A., Mijnarends P.E., Schut H. and Delplancke, M.P., Thin Solid Films 241 (1994) 171-174.

[13] Fink J., Scheerer B., Wernet W., Monkenbusch M., Wegner G., Freund H.-J. and Gonska H., Phys. Rev. B: Condens. Matter. 34 (1986) 1101-1115.

[14] Su D.S., Swoboda M. and Zeitler E., Ultramicroscopy 53 (1993) 97-100.

[15] Raether H.: Excitation of Plasmons and Interband Transitions by Electrons (Springer Berlin Heidelberg New York, 1980).

[16] Schäfer J. , Rinstein J. and Ley L., J. Non-Cryst. Solids 164-166 (1993) 1123-1126.

[17] Ullmann J., Weber A. and Falke U., Mat. Res. Soc. Symp. Proc. 316 (1994) 911-916.

[18] McKenzie D.R., Muller D. and Pailthorpe, B.A., Phys. Rev. Lett. 67 (1991) 773-776.

[19] Batson P.E. and Silcox J., Phys. Rev. B 27 (1983) 5224-5239.

[20] Egerton R. F.: Electron energy-loss spectroscopy in the electron microscope (Plenum Press, New York, 1986).

[21] Daniels J., Festenberg C.V., Raether H. and Zeppenfeld K.: Springer Tracts Mod. Phys. 54 (1970) 78.

[22] Büchner U., Phys. Status Solidi (b); 81 (1977) 227.

[23] Bose S.K., Winer K. and Anderson O.K., Phys. Rev. B 37 (1988) 6262. 